\title{
Crowdsourced Web Engineering and Design
}

\author{
Michael Nebeling, Stefania Leone, and Moira C. Norrie \\ Institute of Information Systems, ETH Zurich, \\ CH-8092 Zurich, Switzerland \\ \{nebeling, leone, norrie\}@inf .ethz.ch
}

\begin{abstract}
We present an approach for the lightweight development of web information systems based on the idea of involving crowds in the underlying engineering and design processes. Our approach is designed to support developers as well as non-technical end-users in composing data-driven web interfaces in a plug-n-play manner. To enable this, we introduce the notion of crowdsourced web site components whose design can gradually evolve as they get associated with more data and functionality contributed by the crowd. Hence, required components must not necessarily pre-exist or be developed by the application designer alone, but can also be created on-demand by publishing an open call to the crowd that may in response provide multiple alternative solutions. The potential of the approach is illustrated based on two initial experiments.
\end{abstract}

Keywords: Lightweight web engineering, end-user development, crowdsourcing.

\section{Introduction}

Crowdsourcing is currently an important topic in both research and industry. The term was originally coined in an article by Jeff Howe [1] and refers to the idea of outsourcing some kind of task to a larger group of people in the form of an open call. In a software engineering context, it is typically used to refer to crowdsourced systems [2] which provide a kernel application that other developers, or even the increasingly larger community of end-users with programming experience, can complement and extend with new peripheral services and system functionality. Two popular examples in a web engineering context are Facebook 1 and WordPres: 2 where many parts in the form of small applications, plugins and themes are developed by the community. However, there is currently no dedicated support in web engineering tools for making use of crowdsourcing.

By contrast, within the HCI community, recent work has been directed towards crowd-powered systems that aim to embed crowds directly into interfaces as a way of supporting collective problem solving through crowdsourcing und human computation 3. Paid micro-task crowdsourcing markets such as Amazon Mechanical Turk play an important role in enabling this research since

\footnotetext{
1 http://www.facebook.com

2 http://www.wordpress.org

3 http://mturk.com
} 
studies established it as a viable platform for crowdsourcing experiments [4]. Hence, our goal is to build on these ideas and bring them to the web engineering community.

Given the proliferation of powerful Web 2.0 platforms such as WordPress and advanced mashup tools, e.g. [5]6, end-users are increasingly provided with support for building web information systems in a plug-n-play manner. However, there is relatively little support if the required web site components do not already exist somewhere on the web. Therefore, we decided to investigate a systematic approach to engineering web applications based on the mashup paradigm even if they are designed from scratch. At the same time, the goal is to support the whole range from less experienced end-users to expert web developers as well as the complete design-build-evaluate cycle by providing support for crowdsourcing at all stages of design. This work builds on previous research on a crowdsourced platform for information system development 78.8 and crowdsourced web site adaptation [9] and extends it towards our proposal for crowdsourced web engineering. In the same sense, it is also related to recent work on crowdsourcing web usability tests before and after deployment 10.

In this paper, we present a first platform and design environment as well as two possible crowdsourcing models for the lightweight composition and crowdsourced design of new web applications based on the proposed paradigm. To facilitate this, our approach also supports the plug-n-play style of composing web applications similar to WordPress and advanced mashup editors. In contrast to these approaches, however, we build on a new notion of crowdsourced web site components that support the evolution of content, presentation and behaviour by continuously refining their design with the help of the crowd. Similar to programmableweb.com or userscripts.org, this may work as a communitybased design approach just by itself, but we also present an integration of our design environment with Mechanical Turk so that crowd workers can directly contribute. The requester can then review and choose from suggested design alternatives to be used in their own applications, as well as starting new iterations based on the best results so far.

Section 2 presents the background to this work. We introduce our crowdsourcing approach along with the platform in Section 3. We then present a first realisation of our proposal in Section 4, followed by the implementation in Section 5 Section 6 reports an initial evaluation of the approach for two simple examples, while concluding remarks are presented in Section 8.

\section{Background}

There are numerous frameworks and development environments for building web information systems. Early approaches have focused on supporting developers in the systematic engineering of web information systems by designing applications at a higher level of abstraction. For example, web methodologies such as WebML [11, UWE [12] and Hera [13] build on a set of different models to describe the structural, navigational and presentation aspects of web information 
systems. Models are typically defined graphically and most methodologies offer a platform for application generation and deployment according to the model definitions. However, while these model-driven approaches are generally very powerful, they follow a bottom-up development method that usually starts from the data model. The focus is therefore on modelling rather than designing which is less appropriate for web designers and non-technical end-users.

In contrast, other research has focused on end-user development of web-based information systems by providing application editors that are closer to a WYSIWYG approach 1415. The WYSIWYG editor presented in 14 supports web information system development using a top-down approach, where a user starts by specifying the presentation layer and creating forms that represent domain entities, from which the underlying system is then generated. However, the presentation is restricted to forms and there is no support for rich UI controls. In [15, a more advanced WYSIWYG visualiser and editor for managing structured datasets based on an active document paradigm is presented. Here, data is stored alongside structure and presentation as an active document, which basically corresponds to a web page.

With the new proliferation of web platforms and services, there has been an increasing trend of building web mashups that integrate and visualise information aggregated from various distributed web data sources rather than designing applications from scratch. In a first response, visual mashup editors such as Yahoo Pipes4, MashMaker [16] and Mash-o-matic [17 have been designed to enable general mashup creation, such as the aggregation of feeds and data visualisation on a map, through the graphical composition and integration of data from different sources. However, while most mashup editors help users integrate information from distributed sources, they do not provide the basic infrastructure to facilitate the design and composition of new applications for their own data, which is one of the scenarios supported by our approach. An exception is MashArt [5] which is a platform that combines the ideas of web information system development and mashups. MashArt enables advanced users to create their own applications through the composition of user interface, application and data components. The focus is on supporting the integration of existing presentation components based on event-based composition, where components can react to events of other components.

Recently, DashMash [6] was introduced as a graphical mashup editor for creating new components based on the same event-based approach, but with increased support for end-users. For example, while composing a system from existing services, DashMash can recommend other services that could also be useful in the current design context. However, it is not clear who defines and configures available services and how the approach can scale to supporting the development of complex web applications. To this end, the work presented in [18] may, in principle, provide an interesting solution based on Firecrow - a web site component extraction tool that can liberate selected interface controls from existing web sites while preserving their functionality. However, the focus of this work is on

4 http://pipes.yahoo.com/pipes/ 
extraction rather than reuse and, due to the complexity and diversity of modern web interfaces, there are many critical edge cases, in particular when it comes to extracting relevant JavaScript code. This approach therefore provides no general solution. In addition, there is currently no specific tool support for reusing and embedding extracted components in existing web pages.

The focus of recent work has been on end-user development approaches and specifically the combination of existing web application components as well as the aggregation of web data from various existing sources. We build on this research, but also take some of the ideas further. The goal is to support application designers in general, which may be non-technical end-users, but also experienced developers, by leveraging crowdsourcing as part of the development process. On the one hand, we envision crowdsourcing scenarios that support end-users by asking the crowd to contribute new web site components with tailored functionality. On the other hand, the crowd may also provide alternative designs or iterate over existing ones, which can support developer creativity and increase quality. In both cases, the idea is that the underlying system functionality and application design can evolve and adapt with the help of crowdsourced contributions. This is in contrast to mashup editors, where the focus lies on service composition rather than refinement and evolution.

\section{Crowdsourced Web Engineering and Design}

Our approach to engineering web information systems with the help of crowdsourcing is based on two aspects. First, we support component-based composition of applications by associating user interface controls with data in a plug-n-play style. Second, we promote a crowdsourcing paradigm for the development, sharing and reuse of web site components. We will first introduce the crowdsourced component concept, followed by the design and composition processes it enables and the underlying crowdsourcing models.

Figure 1 illustrates the component concept. In our approach, we distinguish between the information component part and the user interface component part that together describe an application component. Information components define the data as well as the schema which consists of both structural and behavioural

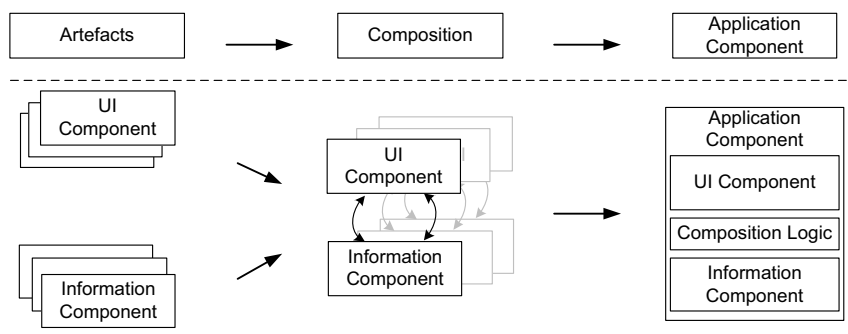

Fig. 1. Crowdsourced Web Site Components Concept 
information. They can therefore be regarded as components that provide data as well as facilities to manage that data [7]. On the other hand, user interface components define static presentation aspects and dynamic user interface behaviour. They can hence be either basic templates for presenting content or more advanced form-based controls and widgets that allow a user to view, work with and manipulate the information.

Further, the processes of designing interface components and linking them to information components are supported from within a design environment that is directly embedded in the web site. As illustrated in Fig. 2a, the process of designing interface components is facilitated by allowing application designers to adapt, compose and group interface elements in order to build more complex ones. For example, in the first step, the designer may choose an image component and associate it with a label to build an image preview. The composition process illustrated in Fig. $2 \mathrm{~b}$ then allows designers to associate the resulting interface components with information. This involves binding the data provided by information components to the interface component. Both processes along with other features of our design tool are described in detail in the next section.

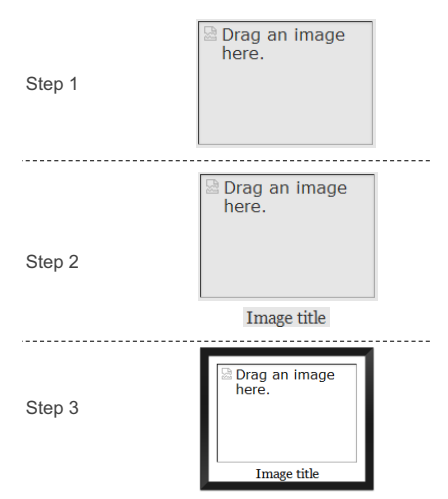

(a) Design Process

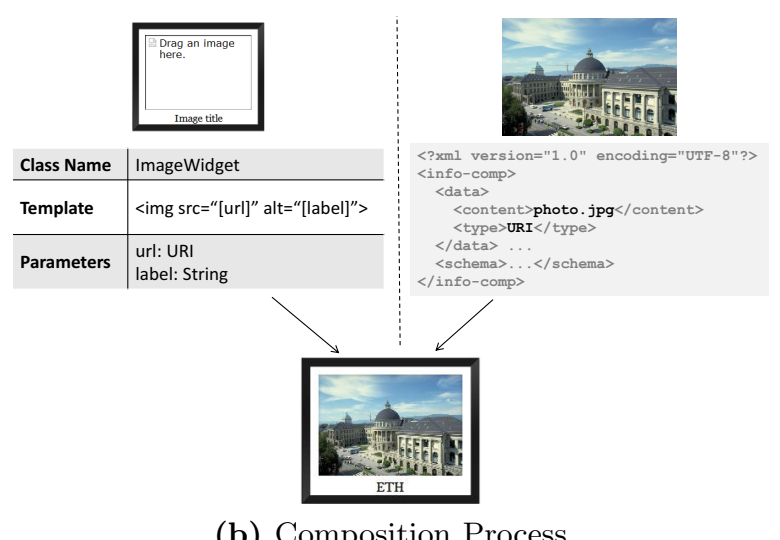

(b) Composition Process

Fig. 2. Development Processes based on Crowdsourced Web Site Components

Moreover, our component concept is designed to support the evolution of components in that both the interface and information component parts can be defined in multiple versions. Here, versions may be alternative solutions or refinements of previous ones [19]. Each of these versions in turn may either be created by the same application designer or be contributed from others through a form of crowdsourcing. Due to the way in which we defined the component concept, the resulting flexibility is reflected at both the information management and user interface levels. There are therefore several possibilities of making use of crowdsourcing based on the concept.

- Crowdsourcing Information Components. Application data as well as the schema that describes the data may be crowdsourced. For data 
crowdsourcing, it is possible to automatically generate a data input user interface based on the component schema to allow crowds to contribute data 20]. Crowdsourcing data is particularly useful for data that is not directly accessible over the web or if existing extraction algorithms are insufficient. These are also two cases where mashup solutions would fail since they require existing interfaces and data sources. Furthermore, it is also possible to crowdsource the schema. One way to do this is through the sharing of schemas from existing applications within a community and enabling their reuse in new applications. This is discussed in detail in [8] and therefore not the focus of this paper. However, since information components define schema and data, both crowdsourcing ideas are enabled by our concept.

- Crowdsourcing Interface Components. Crowdsourcing could also be used in several ways at the user interface design level. For example, non-technical end-users may not be able to develop their own web site components, but could build on those that have been contributed by more experienced developers. At the same time, experienced application developers could benefit from crowdsourcing by getting design feedback and specifically asking for new design proposals. The focus of our previous work in this respect was on increasing the adaptivity of user interfaces by asking end-users to contribute variations of the layout suitable to their particular use context 9 . We build on these techniques, but extend them in two ways. First, to allow for new design ideas that go beyond customisation of existing user interface parts, we developed more flexible tools that enable application designers to add new interface elements or change and replace existing ones with alternatives. Second, we designed a simple means of supporting the definition of new user interface components from scratch. To enable this, a placeholder component is provided that the application designer can specify up to a certain level and which may then be realised and refined through crowdsourcing. Note that placeholder components may be used at the presentation as well as the content level in order to let both aspects of an application component evolve. However, in the scope of this paper, we focus on the idea of crowdsourcing interface rather than information components.

As indicated in previous examples, our approach supports two different crowdsourcing models that complement each other.

- Sharing and Reuse. The first is based on the idea of building a common component library to enable the sharing and reuse of components within a community. Given the component concept, it is possible to share either information or interface components, or both, and this with or without data. This allows application designers to contribute as well as benefit from shared components within the community.

- Active Crowdsourcing. The second model is based on services provided by paid crowdsourcing markets like Mechanical Turk. This then turns the previous, indirect crowdsourcing model into an active request-response cycle that enables the application designer to directly call on the crowd by 
publishing requests to the crowdsourcing service. Moreover, this model also gives control over additional parameters such as the number of workers to contribute to the design process as well as time and cost.

The two models are complementary since both can contribute to having a larger library of shared components. While this is obvious in the first case, also in the latter case it can be supported by sharing crowdsourced components again with the community. The models can therefore work independently, but may more effectively be combined by leveraging both the application developer community as well as involving paid, external crowds.

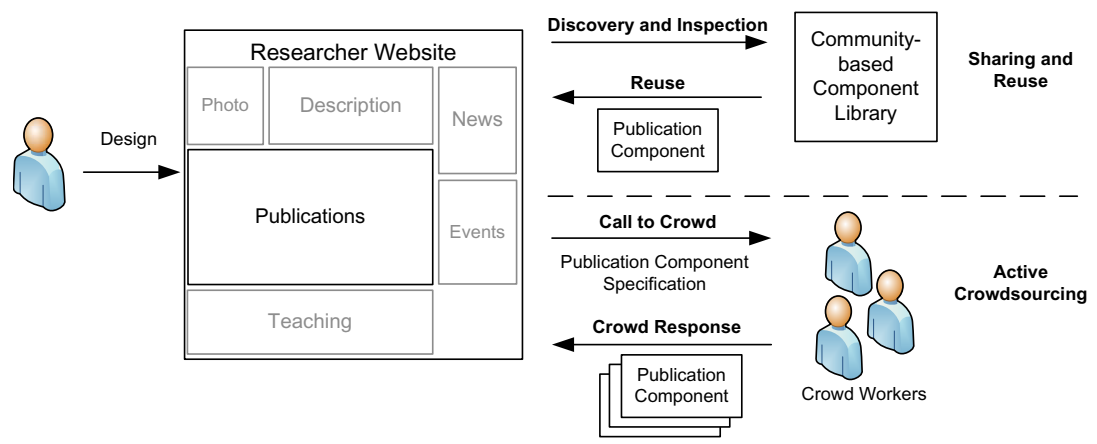

Fig. 3. Crowdsourcing Models illustrated for an Example Web Site

Figure 3 summarises the ideas behind our crowdsourced web engineering approach and illustrates them for the scenario of creating a researcher web page. In the example, the application designer decides to use the crowd for the design of a publications component and may choose from two complementary crowdsourcing models. On the one hand, the application designer may access the communitybased component library and search for a suitable component to be reused. On the other hand, the designer may issue an open call to the crowd and select a component from the crowd response. Application designers are encouraged to combine the two models and share components received through the open call in the community-based component library.

\section{Crowdsourcing Platform and CrowdDesign Prototype}

For crowdsourced web application development based on the new component concept and the crowdsourcing models presented in the previous sections, we have developed a crowdsourcing platform and web-based design environment, CrowdDesign. The underlying architecture is illustrated in Fig. 4,

The platform is responsible for orchestrating the definition and sharing of both information and interface components. Likewise, two separate libraries of shared components, one for information components and one for interface components, are maintained by the platform. Application designers can access these 


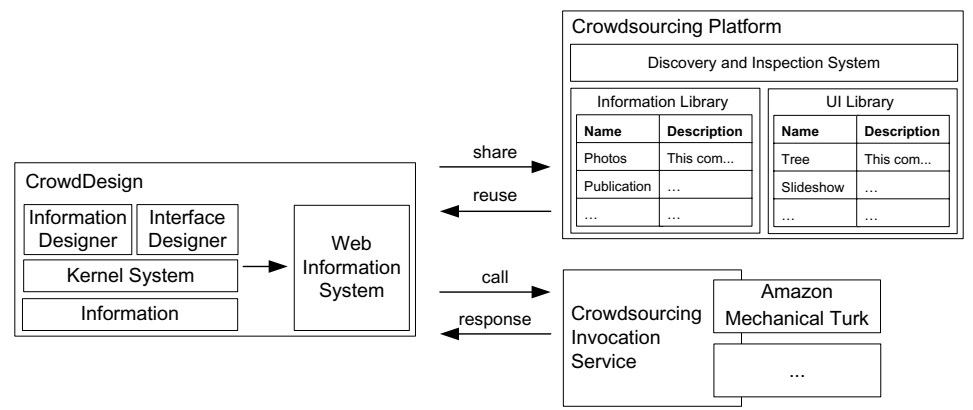

Fig. 4. Architecture

libraries either directly through the discovery and inspection system offered by the platform, or transparently through the development environment when designing applications. Each application designer contributing to the aforementioned crowdsourcing scenarios is provided with a local CrowdDesign instance running on the client machine. The platform services are exposed through the visual design environment of CrowdDesign that is composed of two parts. The first part is an information designer that supports the definition of information components as presented in [8]. The second is an interface designer for the customisation of existing components and the definition of new interface components. The design tools therefore enable the design process in terms of definition and maintenance of instances of each component type as well as their composition to application components. Web applications based on our approach are directly hosted by the platform and run from within CrowdDesign, but they can also be deployed separately as standalone web information systems using the same kernel system. The integration with Mechanical Turk is facilitated by the crowdsourcing invocation service as an intermediary which may be extended with support for other crowdsourcing markets.

The CrowdDesign prototype is divided into several different areas shown in Fig. 5. The client area in the centre is the workspace where application designers can design and compose applications. On the left, the designer has access to the library of user interface components shared within the community. As mentioned previously, these may include basic user interface form controls, such as lists and buttons, as well as more complex widgets such as image slideshows or video players. On the right, the application designer has access to their own or shared information components that are organised by type and can be browsed and accessed via a hierarchical navigation.

The application designer initiates the actual composition process by simply dragging components from the information library and dropping them on interface elements in the workspace. CrowdDesign then performs an automatic mapping between the user interface component and the information component depending on supported parameters and the data type. For example, a picture view component may define two parameters, namely a URL for the image and text for the label. The application designer can then associate text components 


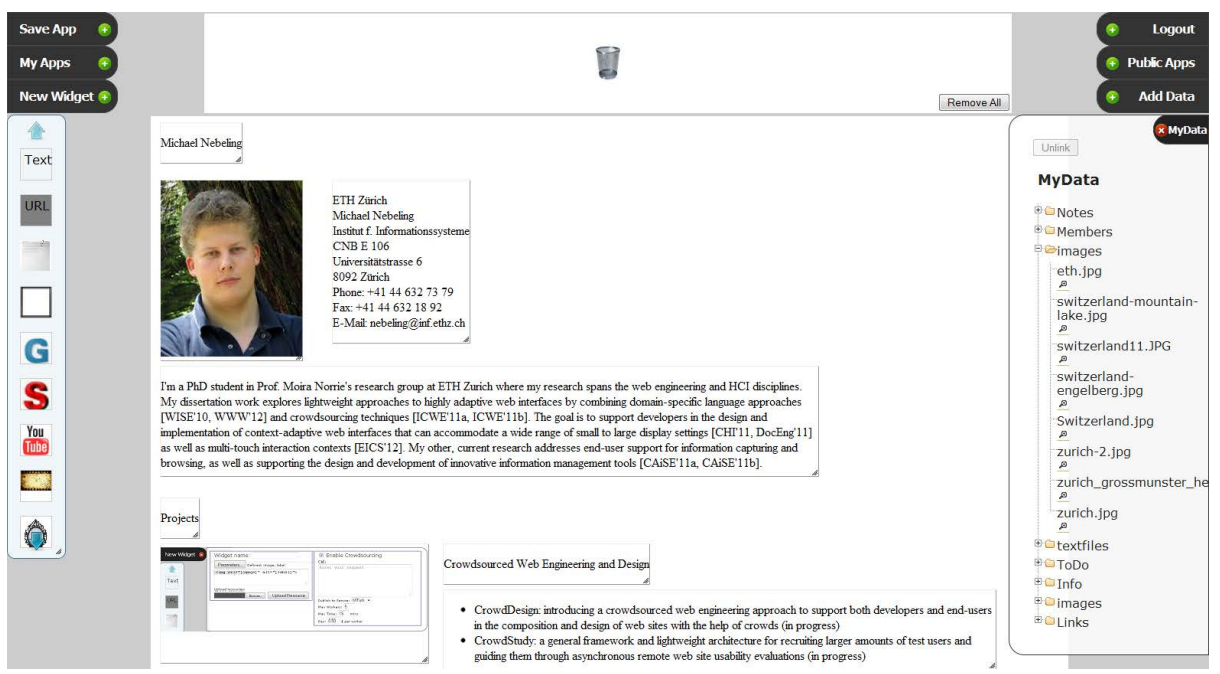

Fig. 5. CrowdDesign Tool

with the picture view in order to set the label as well as an image URL to link to the actual image. For some widgets such as the image slideshow from previous examples, it is also possible to associate a collection of images with the control in a single interaction if the underlying presentation template provides support for array parameters. Note that the application designer can also customise and edit the suggested mapping in a separate dialog (not shown here), which is usually optional, but may be required in the case of ambiguities.

In our current prototype, we use a rather simple representation of both interface and information components. Both types of components are identified by a component descriptor. Interface components are represented as a parametrised template that defines presentation as well as behaviour using a combination of HTML, CSS and JavaScript. Information components define the content in the form of semi-structured data in XML format. Application designers may simply add new information components by uploading corresponding XML files. New interface components can be defined programmatically and imported into the workspace. As shown in Fig. 6, this is supported by allowing application designers to specify supported parameters and the corresponding presentation template as well as uploading required resource files. On the other hand, interface components from the interface library can be added to the workspace via drag-n-drop. Workspace components can be resized and positioned freely as required. Alternatively, they may also be anchored at the edges of other components in the interface. The difference is that, in the first case, the position and size within the workspace are only defined in terms of CSS, while in the latter, CrowdDesign also manages the underlying HTML DOM tree to maintain a fully functional web interface. This is based on the techniques presented in $[9$.

In addition, the application designer can formulate an active crowdsourcing request by providing a description of the task and desired result for a new 


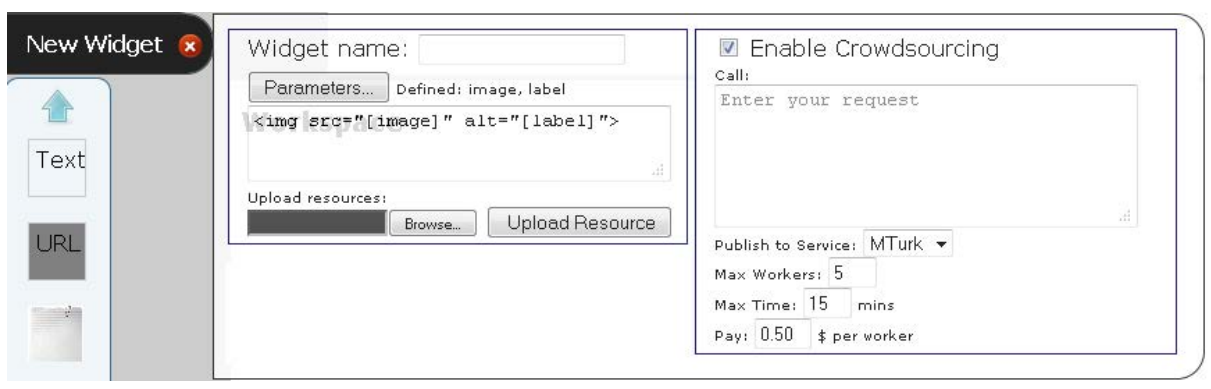

Fig. 6. New Widget Dialog and Simple Crowdsourcing Interface

interface component. Alternatively, the designer may formulate a task based on an existing component. In both cases, a placeholder is first used in the workspace, while CrowdDesign can automatically submit requests to Mechanical Turk on behalf of the application designer and periodically check for responses. For the integration with Mechanical Turk, the application designer needs to specify additional parameters such as the number of tasks to be generated, maximum work time and the payment to be awarded for each response the application designer accepts after review.

On Mechanical Turk, workers interested in CrowdDesign tasks are then first shown the task description and any other information provided by the application designer. Should they choose to accept the task, they are forwarded to a CrowdDesign sandbox providing access to selected parts of the workspace and the tools necessary to contribute to the task. By default, CrowdDesign is configured to show both the interface and information components that are relevant and to allow for customisation of the existing parts as well as adding new components using the aforementioned CrowdDesign features. As shown in Fig. 7a responses collected from workers can be previewed by the application designer who may accept or reject the results after exploring them in more detail. Each accepted contribution is automatically imported into the application designer's local CrowdDesign workspace and payment will be issued to the respective worker via the Mechanical Turk platform.

Note that workspace components can be removed simply by dragging them onto the trash bin shown in the top area of Fig. 5. This supports the idea of rapidly prototyping new components by building on relevant parts of existing ones. It is therefore also possible to merge and benefit from several crowd solutions. Likewise, components may again be recycled from the bin. Moreover, only the link between selected interface and information components may be revoked by clicking the unlink button.

Finally, application designers can save the state of the workspace so that it can be reaccessed in later sessions. Each workspace state is described by an additional XML manifest file and versioned separately together with the respective component descriptors. Further, the current workspace may also be exported as a new user interface component that will then appear in the interface library. 


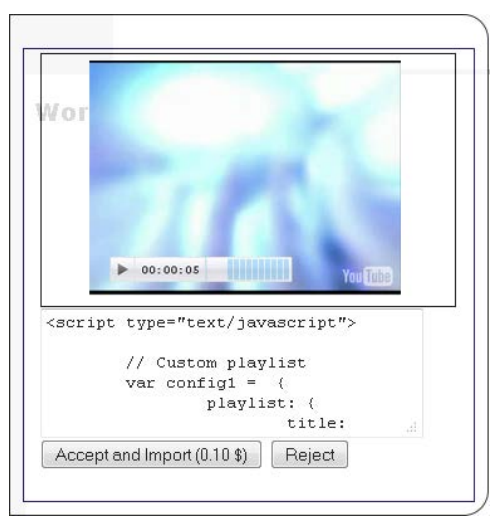

(a) Crowd Response Dialog

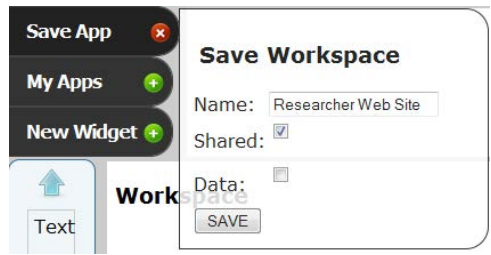

(b) Application Sharing

Fig. 7. Features for Reviewing and Sharing of Components

This can be done locally for the application designer to reuse the component in other applications or globally within the community [8. As illustrated in Fig. 7b, we offer the possibility of exporting and sharing new user interface components either with or without data. In the case that data is shared, the user interface component is shared together with the associated information components as a snapshot so that all parts can be reused independent of how the source components may evolve as part of other operations.

\section{Implementation}

The community platform is implemented on the server-side using PHP based on the open-source CakePHP framework 5 in combination with a MySQL database. On the other hand, our web site design tool, CrowdDesign, is implemented using client-side technologies HTML, CSS3 and JavaScript based on the popular jQuery framework6. Much of the interface design functionality is based on the implementation of [9], while the information designer is currently simplified to a basic visual data explorer building on AJAX techniques to fetch data on demand as well as PHP's support for file uploads of new information components specified in XML.

The integration with Mechanical Turk is based on the MTurk API to generate and submit new component-related tasks, retrieve completed work, and approve or deny that work. Instead of building on MTurk's in-built questionresponse API, we use an external question in order to wrap the developer's request. CrowdDesign can be configured to be either embedded into an HTML iframe or opened in a new window. To keep a record of workers that contributed

\footnotetext{
5 http://cakephp.org

6 http://jquery.com
} 
new components, we use MTurk's internal task assignment code to identify each CrowdDesign sandbox as well as associating it with the corresponding component versions to coordinate the import into the respective CrowdDesign instance.

\section{Evaluation}

We evaluated the basic functionality of CrowdDesign in two smaller experiments. The first experiment was conducted with the help of members of our group and designed to simulate a community-based design approach. In this experiment, one of the members got the task of designing a web page featuring his research profile together with the help of others. The other members got the task of browsing web pages of researchers and extracting common components and sharing them in the library. To keep the experiment simple, screenshots rather than fully-functional components were sufficient.

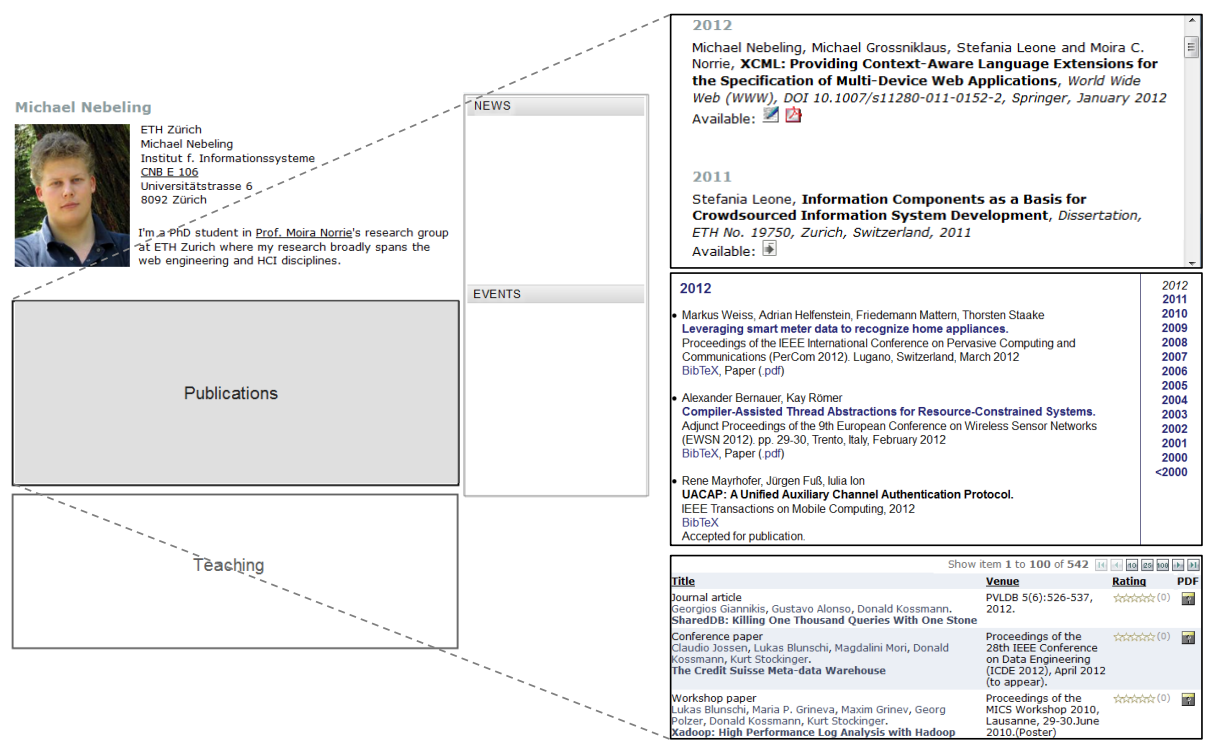

Fig. 8. Intermediate Design and Selected Community Answers

Figure 8 shows a screenshot of the workspace with an intermediary design of the researcher's homepage. At this stage, the application designer structured the page into several components. Some of the components were rapidly prototyped using his own data, such as the photo, biography and contact details, while others, such as the news and events components, have only been sketched using labelled placeholder components. In the centre, the application designer started to design a component for listing his publications. Five members of our group participated in this experiment and some of them contributed several components with different designs and functionality as input for developing such a publication component. 
Also shown in the figure are three of the crowdsourced publication components originating from web sites of research groups in our institute. While all components list the publication details as well as a link to an electronic copy of each publication, there are also several differences that the designer may take into account for developing his own component. For example, the first crowdsourced publication component lists publications in reverse chronological order grouped by year and users can scroll through the list. In contrast, the solution in the middle displays publications only for a selected year and a bibtex entry is also provided. The last solution paginates the list of publications and provides navigation controls similar to database browsers. An additional functionality in this solution is that publications can also be rated.

In the second experiment, we used an active crowdsourcing model and instead built on Mechanical Turk. Here, the general task was to design a new search component for finding content within a web page. For this experiment, we issued a call for an interface component in which we specified that it should present a simple search interface and include the necessary JavaScript to run the query. The call was issued via Mechanical Turk with a 15 minute request for 5 workers at 50 cents each. Workers were presented a minimal CrowdDesign interface to upload the required resources and enter the necessary code. Figure 9 shows three of the answers we received in response. The first two are rather simple form interfaces making use of only HTML. While they both catered for basic search functionality using JavaScript, the code provided by the crowd only worked for certain browsers. The third is a solution that relies on an open-source JavaScript plugin that is cross-browser compatible. Moreover, the interface uses a more sophisticated popup-based design and also allows to search backwards.
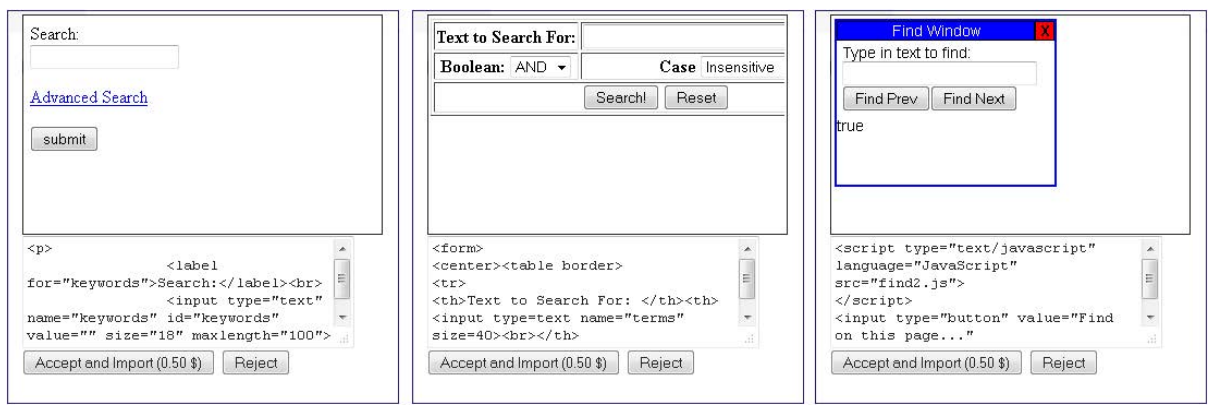

Fig. 9. Worker Responses for Simple Search Component Experiment

\section{Discussion}

Even though our evaluation is still preliminary, it already showed that our new idea of crowdsourced web engineering and design is generally fruitful and that a system like CrowdDesign could facilitate the task of crowdsourcing the design of new application components. While our first experiment targeted a communitybased design approach, it still left a single developer in charge. However, with 
the ideas and tools presented in this paper, it is in principle possible to enable the co-development of components and to allow multiple application designers to work on and develop for the same web site. This would then provide a first basis for additional ideas of participatory design. The second experiment provided first insights into the different roles crowdsourcing could play for the proposed approach. As mentioned previously, it can be used both to support non-technical users to develop complex application components by building on the contributions provided by others. At the same time, it could allow even advanced developers to benefit from the design input of other users and generate new ideas. Generally, it is important to see how our use of crowdsourcing allows us to overcome some of the limitations of previous approaches. For example, content extraction is typically found to be difficult given that most existing components on the web are tightly woven into the hosting web sites and rely on a combination of HTML, CSS and JavaScript. While there are some tools such as Firecrow [18] that can be used to extract interface controls from existing web sites, it is still hard to fully automate the extraction process and also difficult to support the integration with new web sites. We argue that our approach based on crowdsourcing principles can provide a solution for such problems since experienced developers can do the extraction and package the components in such a way that they can easily be integrated with new web sites. Platforms such as programmableweb.com that are maintained by an active community show that this is not only feasible in principle, but also has the ability to scale in practice.

To enable future experiments with such ideas, we plan on making the tool and platform proposed in this paper available to the community. This would then also allow other researchers to explore the ideas of collaborative web design and to leverage crowdsourcing for hard problems such as component extraction from existing web sites.

\section{Conclusion}

We have presented an approach for crowdsourced web engineering and design as well as the enabling concept, platform and design environment. Our focus has so far been on the architectural and tool support. The supported crowdsourcing scenarios naturally make the approach dynamic and scalable. As a consequence, a number of issues may emerge, such as motivational incentives to contribute, quality control and security mechanisms as well as general questions of authorship and credit. These could be investigated based on our proposal and the first realisation presented in this paper. At the same time, we plan to extend and explore the presented techniques mainly along two directions. First, we plan to foster the engineering aspects of our approach and find ways of improving the technical quality of crowdsourced contributions. Second, we will investigate new possibilities enabled by our approach such as support for creativity and participatory design.

Acknowledgements. We would like to thank our IS Lab 2011 students and Kazuhiro Komoda for their help with the implementation of the prototype presented in this paper. 


\section{References}

1. Howe, J.: The Rise of Crowdsourcing. Wired 14(6) (2006)

2. Kazman, R., Chen, H.M.: The Metropolis Model: A New Logic for Development of Crowdsourced Systems. CACM 52(7) (2009)

3. Quinn, A.J., Bederson, B.B.: Human Computation: A Survey and Taxonomy of a Growing Field. In: Proc. CHI (2011)

4. Kittur, A., Chi, E.H., Suh, B.: Crowdsourcing User Studies With Mechanical Turk. In: Proc. CHI (2008)

5. Daniel, F., Casati, F., Benatallah, B., Shan, M.-C.: Hosted Universal Composition: Models, Languages and Infrastructure in mashArt. In: Laender, A.H.F., Castano, S., Dayal, U., Casati, F., de Oliveira, J.P.M. (eds.) ER 2009. LNCS, vol. 5829, pp. 428-443. Springer, Heidelberg (2009)

6. Cappiello, C., Matera, M., Picozzi, M., Sprega, G., Barbagallo, D., Francalanci, C.: DashMash: A Mashup Environment for End User Development. In: Auer, S., Díaz, O., Papadopoulos, G.A. (eds.) ICWE 2011. LNCS, vol. 6757, pp. 152-166. Springer, Heidelberg (2011)

7. Leone, S., Geel, M., Norrie, M.C.: Managing Personal Information through Information Components. In: Soffer, P., Proper, E. (eds.) CAiSE Forum 2010. LNBIP, vol. 72, pp. 1-14. Springer, Heidelberg (2011)

8. Leone, S., Norrie, M.C.: Constructing eCommerce Systems from Shared MicroSchemas. In: Proc. CoopIS (2011)

9. Nebeling, M., Norrie, M.C.: Tools and Architectural Support for Crowdsourced Adaptation of Web Interfaces. In: Auer, S., Díaz, O., Papadopoulos, G.A. (eds.) ICWE 2011. LNCS, vol. 6757, pp. 243-257. Springer, Heidelberg (2011)

10. Nebeling, M., Speicher, M., Grossniklaus, M., Norrie, M.C.: Crowdsourced Web Site Evaluation with CrowdStudy. In: Brambilla, M., Tokuda, T., Tolksdorf, R. (eds.) ICWE 2012. LNCS, vol. 7387, pp. 494-497. Springer, Heidelberg (2012)

11. Ceri, S., Fraternali, P., Bongio, A., Brambilla, M., Comai, S., Matera, M.: Designing Data-Intensive Web Applications. Morgan Kaufmann Publishers Inc. (2002)

12. Hennicker, R., Koch, N.: A UML-Based Methodology for Hypermedia Design. In: Evans, A., Caskurlu, B., Selic, B. (eds.) UML 2000. LNCS, vol. 1939, pp. 410-424. Springer, Heidelberg (2000)

13. Vdovják, R., Frăsincar, F., Houben, G.J., Barna, P.: Engineering Semantic Web Information Systems in Hera. JWE 1(1-2) (2003)

14. Yang, F., Gupta, N., Botev, C., Churchill, E.F., Levchenko, G., Shanmugasundaram, J.: WYSIWYG Development of Data Driven Web Applications. PVLDB 1(1) (2008)

15. Karger, D.R., Ostler, S., Lee, R.: The Web Page as a WYSIWYG End-User Customizable Database-backed Information Management Application. In: Proc. UIST (2009)

16. Ennals, R., Brewer, E., Garofalakis, M., Shadle, M., Gandhi, P.: Intel Mash Maker: join the web. SIGMOD 36(4) (2007)

17. Murthy, S., Maier, D., Delcambre, L.: Mash-o-Matic. In: Proc. DocEng. (2006)

18. Maras, J., Stula, M., Carlson, J.: Reusing Web Application User-Interface Controls. In: Auer, S., Díaz, O., Papadopoulos, G.A. (eds.) ICWE 2011. LNCS, vol. 6757, pp. 228-242. Springer, Heidelberg (2011)

19. Grossniklaus, M., Norrie, M.: An Object-Oriented Version Model for ContextAware Data Management. In: Benatallah, B., Casati, F., Georgakopoulos, D., Bartolini, C., Sadiq, W., Godart, C. (eds.) WISE 2007. LNCS, vol. 4831, pp. 398-409. Springer, Heidelberg (2007)

20. Franklin, M.J., Kossmann, D., Kraska, T., Ramesh, S., Xin, R.: CrowdDB: Answering Queries with Crowdsourcing. In: Proc. SIGMOD (2011) 